\title{
INDUSTRIALS SIMULATION MODELING TECHNOLOGY APPLICATION TO IMPROVE THE EFFICIENCY IN AUTOMATIC PRODUCTION LINE EQUIPMENT
}

\author{
Tuan-Linh Nguyen*, ORCID ID: 0000-0002-1960-9794 \\ Department of Mechanical Engineering, Hanoi University of Industry, Hanoi City,Vietnam \\ *Corresponding author: Tuan-Linh Nguyen, nguyentuanlinh@haui.edu.vn
}

Received: 06. 12. 2021

Accepted: 08. 06. 2021

\begin{abstract}
Currently, the application of technical advances to production plays an important role in improving productivity and saving production costs. The techniques applying information technology bring high efficiency, accuracy, reliability, pre-assessment of the results. Modeling and simulation are method that are widely used from research, design, manufacturing to operate the systems. With the help of computers, with high computational speed and large memory, the modeling method was strongly developed, bringing great efficiency in research and production practice. The assessment of effective use of equipment in the production line has a decisive role in increasing the productivity and decreasing cost. Therefore, the analysis and evaluation of the production line by the simulation model method is highly practical, bringing many effects in the management and use of equipment. In this study, a simulation of a specific problem was performed to estimate the simulated workshop. Then, building a new plan and comparing the proposed plane with the original plan to provide the reasonable solutions for the production process to effectively use the equipment in the production line.
\end{abstract}

Keywords: modeling and simulation, effective use, equipment, automatic production line.

\section{Introduction}

Today it is possible to see that all areas of human activity use the method of modeling and simulation at different levels. This is especially important in the realm of controlling technical and social systems because control is the process of acquiring information from the system, identifying the system according to a certain pattern and delivering decide appropriate to control the system [1]. This process is continued continuously to bring the movement system to a predetermined goal. Through modeling and simulation, we can analyze, study complex systems, determine the operating characteristics and behaviors of the systems. The simulation results are used to design, manufacture as well as determine the operating mode of the system. From there, thanks to the method of modeling and simulation, it is possible to give many scenarios to choose the optimal plan. The simulation model can be used in the following 4 categories:

- Device explanation to identify the system or problem.

- Analysis to identify elements, components and events related to the system. 
- Inspect the general design and evaluate proposed solutions.

- Predict and assist in future development planning.

Nowadays, besides the aforementioned method, the simulation method is strongly developed and widely applied. Models that are built on a simulation method are called simulation models or numerical models. Simulation method allows to bring into the model many factors close to reality. Simultaneously, the model is solved on computers with fast calculation speed and large capacity, so the results obtained are highly accurate [2]. Therefore, the simulation method has created conditions to solve complex problems such as modeling problems with large, random, and nonlinear systems with time-varying parameters [3]. The simulation method is especially effective when it is necessary to model large systems whose basic feature is a hierarchical structure, a subsystem structure, between subsystems and the control center exchange information with each other. Simulation method is also effective when modeling systems with random elements, inadequate information, information will be added during simulation, during the information exchange between people controls with objects $[4,5]$. Simulation method is applied to model in many fields such as engineering, economics, society, biology, especially large, complex systems, etc. with many random affecting factors [6]. In addition, the application of artificial intelligence and neural networks is a development direction for technology solutions [7].

In the system design stage, modeling helps the designer to select the structure and parameters of the system to synthesize the system. At the fabrication stage, modeling helps to choose materials and fabrication technology. At the system operation stage, modeling helps the operator solve the optimization problems, predict system states.

Especially in the case of combining the expert system with modeling method, it can solve many control problems, save time as well as material and financial costs.

\section{Material and Methods}

The production system includes many functional subsystems such as supplying materials, energy, processing, and processing; assembling and completing products; consumption distribution. The production process control is the control center. The input of the system is the customer's order, the output of the system is the final product.

From the figure 1 , we see that in a control system, there are many elements commonly called entities, each with its properties. A process that causes a change in the system is called an activity. An action that changes the system's state is called an event. The set of variables that reflect the state of the system at a time is called state variables.

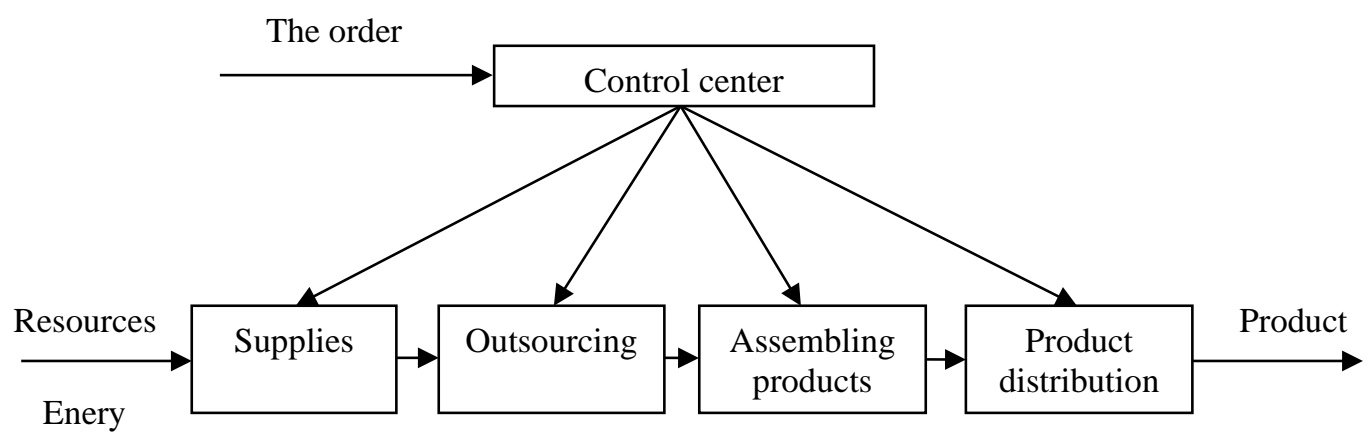

Figure 1. Production process control system. 
There are two methods of studying the system: research on the real system and research on its substitution model. It is clear that research on the real systems gives honest and objective results. However, in many cases conducting research on real systems faces many difficulties, so the best and most convenient method is to study on its model. That is why the modeling method is focused on research and development and this method plays an important role in the development of science and technology [8].

The modeling process is carried out as follows. Call the simulated system $\mathrm{S}$. The first step is to model the system $S$ with its internal relationships. For convenience in modeling, we often divide the $S$ system into several subsystems according to certain criteria $S=S_{1}, S_{2}$, .... $S_{i} \ldots, S_{n}$. Next, mathematically describe the subsystems and their relationships. Usually there is a relationship of energy exchange and information exchange between subsystems. The second step is to model the surrounding environment $E$, where the system $S$ works, with the interactive relationships between $\mathrm{S}$ and $\mathrm{E}$. Once the model of $\mathrm{S}$ and $\mathrm{E}$ is available, conducting the above experiments model, i.e., for $S$ and $E$ to work in a certain condition. The result is a set of system parameters, often referred to as the determination of a system work point. These experiments were repeated many times and the simulation results were evaluated by statistical probability. The simulation results are more accurate if the number of experiments, also known as simulation steps, is larger. In theory the simulation step are finite but must be large enough and depends on the requirement of accuracy.

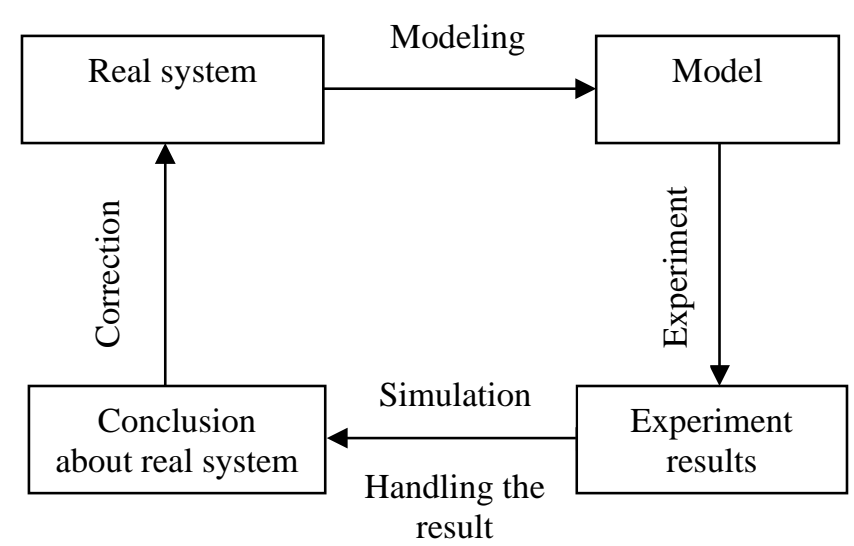

Figure 2. The process of research by simulation method.

When conducting simulation studies, it is often performed the following steps:

Step 1: Develop simulation goals and research plans

The first is to clearly define the simulation research objective. That goal is represented by evaluation criteria, by a system of questions that need to be answered.

Step 2: Collect data and define the principal model $M$

Depending on the simulation goal, we collect the information and corresponding data of $\mathrm{S}$ system and $\mathrm{E}$ environment. On that basis, we build the principal model $\mathrm{M}$. The principal model that is the anti-mathematical model reflect the nature of the S-system.

Step 3: Validate the principal model

Validation of the model principal is to check the model's validity. A principal model must reflect the true nature of system $S$ and $E$ environment but at the same time must not be too complicated and cumbersome. If the principal model $M$ fails to meet the requirements, it is necessary to collect more information and data to rebuild the model. 
Step 4: Build an M simulation on the computer

Mmp simulation models are programs that run on computers also known as numerical modeling or simulation models. These programs are written in common languages such as FORTRAN, PASCAL, $\mathrm{C}++$ or specialized languages for simulation such as GPSS, SIMSSCRIPT, SLAM II, SIMPLE ++, and so on.

Step 5: Test run.

After installing the program, run the test to see if the simulation model correctly reflects the system $\mathrm{S}$ and $\mathrm{E}$ environment characteristics. At this stage also fixes programming errors.

Step 6: Verify the simulation model.

After the test run, it is possible to verify and evaluate whether the simulation model is satisfactory or not, if not, go back from step 2.

Model validation and validation are two important procedures for verifying that the built model should be usable or not. Verification is to check whether the programming is correct, the computer program can run, the input and output data is convenient and accurate or not. Validation of the model is to evaluate whether the model reflects the nature of the real system or not, whether the simulation results meet the research requirements.

Step 7: Planning the simulation experiment

In this step some simulation conditions must be defined. The first is to determine the first condition, the final condition, or the simulation length. Next to determine the number of tests, also known as the number of independent simulation runs. For the simulation data to be completely independent of each other, each run the simulation uses a different random seed. Finally determine the simulation time of each part or whole model. Based on the simulation results (in step 9)

calibrate the experimental plan to get results with the precision required.

Step 8: Simulation experiment

Run the test program according to the built plan in step 7. This is the step to perform the simulation. The results from this step are simulation output.

Step 9: Processing simulation results

Simulation experiments often give lots of data with statistical probability. Therefore, to get the final result with high accuracy, it is required to use statistical probability method to process the output data. These results should be presented in an explicit format conducive to storage and use.

Step 10: Use and store results

Use the simulation results for the intended purpose and archive them as documents that can be used over and over again.

\section{Application of modeling and simulation for automatic production line Requirements of the problem}

When building an automatic production line, the problem is from the requirement of productivity, labor, and equipment to be used effectively to avoid expensive investment or not using up the equipment, machinery and labor. The following is a simulation model that simulates an automatic painting workshop, for example a simulation model of a mobile phone cover coating workshop.

The steps in the model are as follows: 
1. Embryos of the foundry of the foundry have been treated with burr and check for errors and defects and are sent to the paint shop for painting and plating as required.

2. The product will be put into the coating machine under the hanging conveyor. After going through the paint machine, it will be transferred to the drier to dry the product and continue to the aluminum plating machine. Finally, the product will be checked by the worker to remove the defects, if the product has any error, it will be transferred to the drier position for re-implementation. Products are made in the workshop through the steps below.

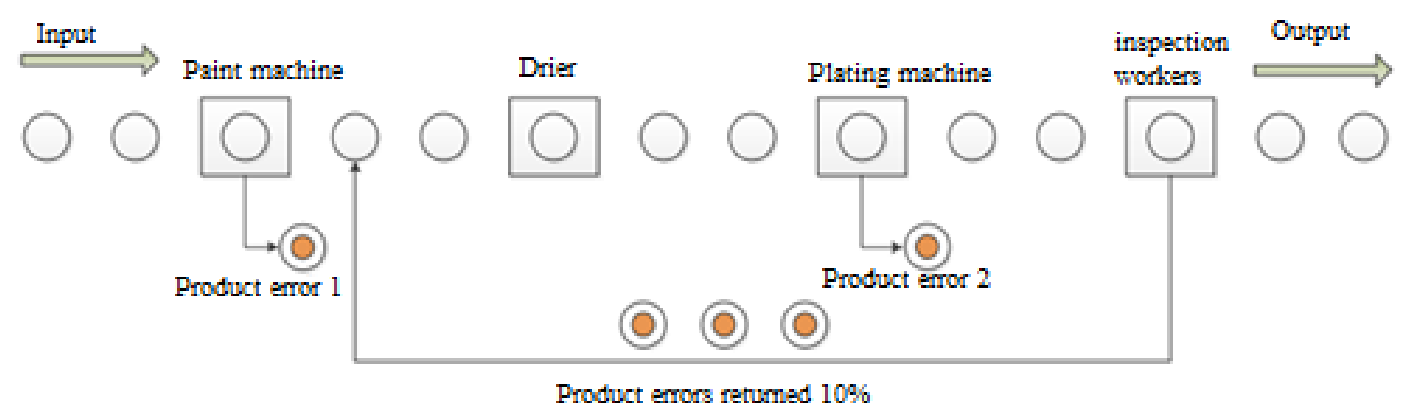

Figure 3. Painting and plating workshop diagrams.

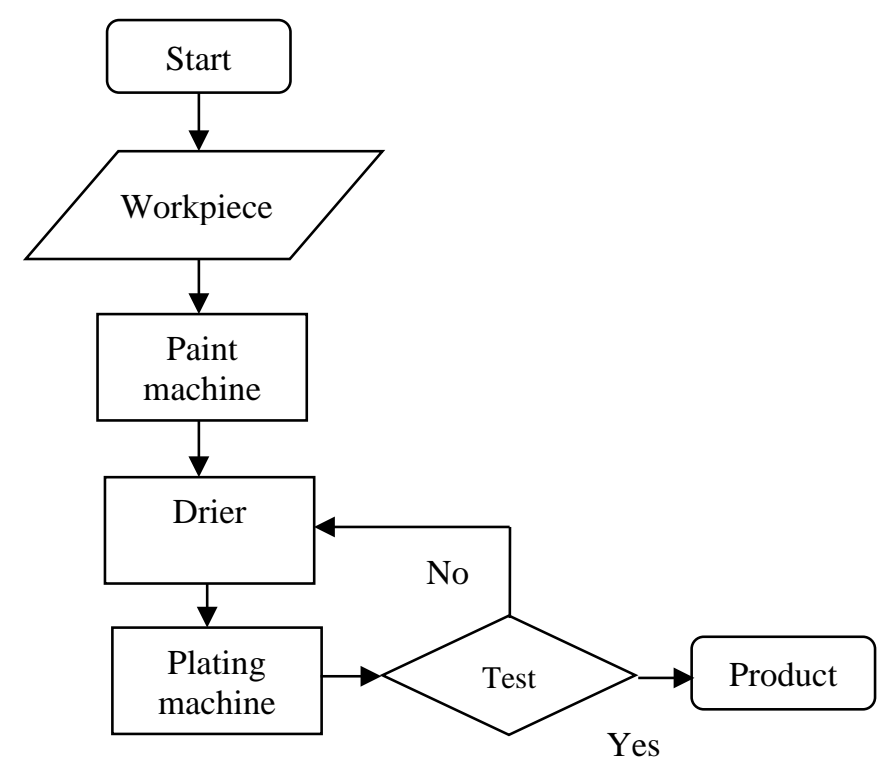

Figure 4. Painting and plating workshop block diagrams.

Computer animation is used for the purpose of judging true or false or to depict the system developed and shown below.

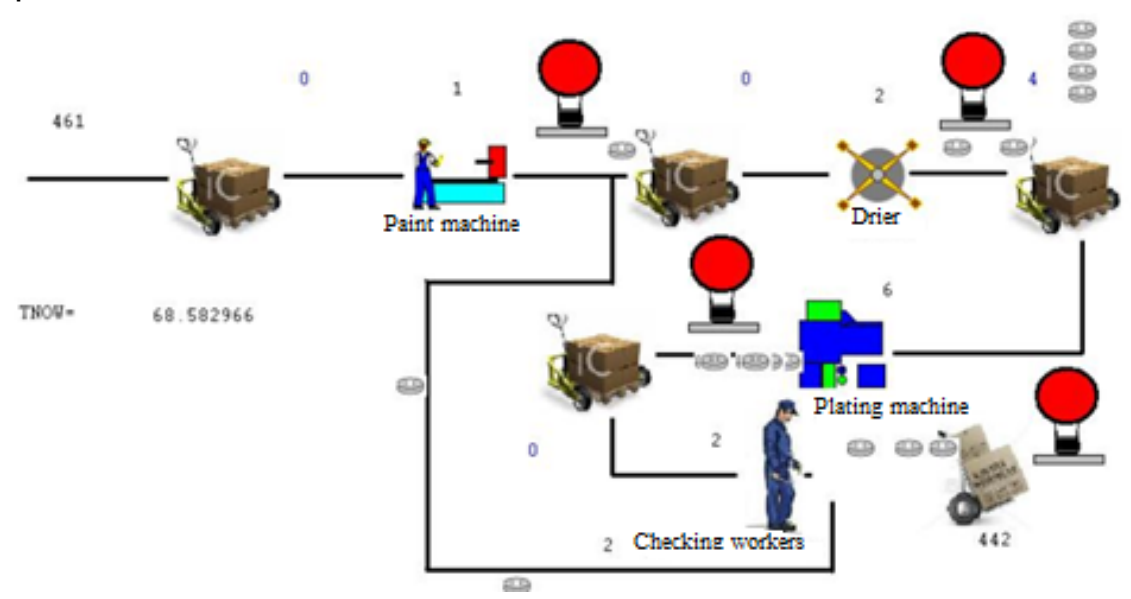

Figure 5. Model of painting and plating workshop scene. 
The operation mode of the workshop is as follows:

1. The plastic billet is taken to the paint machine area to paint the product.

2. They are then transported to the drier for drying.

3. They are then shipped to the chrome plating machine.

4. Finally, they go to the site of the inspector to check the quality of the product surface. If guaranteed, they would be shipped to the next factory. Otherwise, they will be transported back to the drier site for drying or to an aluminum plating machine for rework.

The operation of the system will be simulated under the following assumptions:

1. The arrival times between the two products for the paint machine is exponentially functional with a value of 0.14 minutes.

2. The paint machines operate side by side and the delivery time is a uniform probability between 0.15 and 0.25 minutes.

3. The driers also operate side by side and the delivery time is a uniform probability between 0.25 and 0.35 minutes.

4. The plating machines also operate side by side with uniform distribution time between 0.4 and 1 minute.

5. The inspection workers also work side by side at the test stations. The distribution time as uniform probability is between 0.2 and 0.3 minutes. On average about $90 \%$ meet the requirements and move on to the next workshop. The remaining $10 \%$ of defective products will be sent back to the drier for rework and returned every 10 minutes.

6. Defective products after painting and plating operations are automatically classified. The classification time is 1 minute.

\section{Production line modeling}

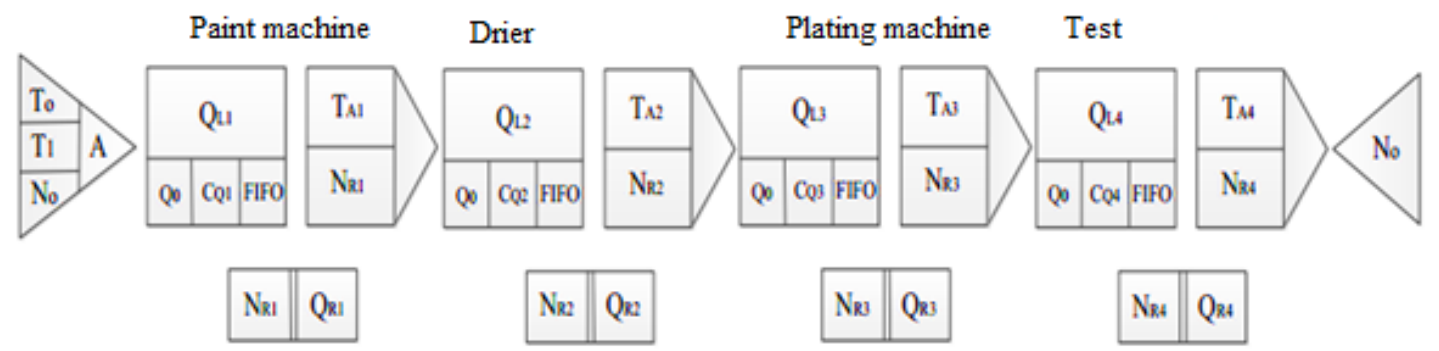

Figure 6. Modeling of the steps of the painting and plating process.

In which:

1. Input (ENTRANCE):

Functions: Simulates incoming objects.

Properties:

T0: Time between next visits; T1: Time of the first arrival of the object.

NO: Maximum number of objects possible through the node.

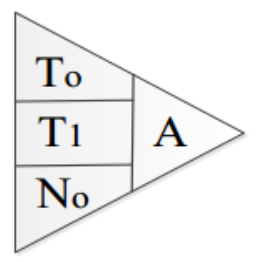

A: Properties of the object. 
2. Queue (QUERY):

Function: Simulate the queue.

Properties:

QL: The current length of the queue; Q0: Recommended queue length.

CQ: The capacity of the queue.

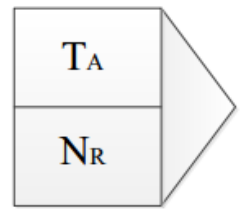

OQ: Service principle: FIFO (first in first out), LIFO (first in, first out), priority.

3. Activities (ACTIVITY):

Function: Simulate serving.

Properties:

TA: Time during the service; NR: Number of sources performing service.

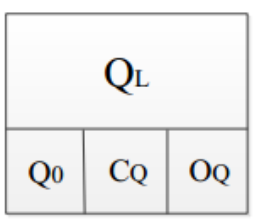

4. Source (RESOURSES):

Functions: Simulating a source is available.

Properties:

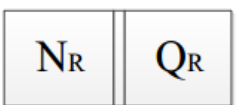

NR: Number of sources; QR: The ability of the source.

5. Ending (EXIT):

Functions: Simulates leaving objects.

Properties:

No: Number of objects leaving the system.
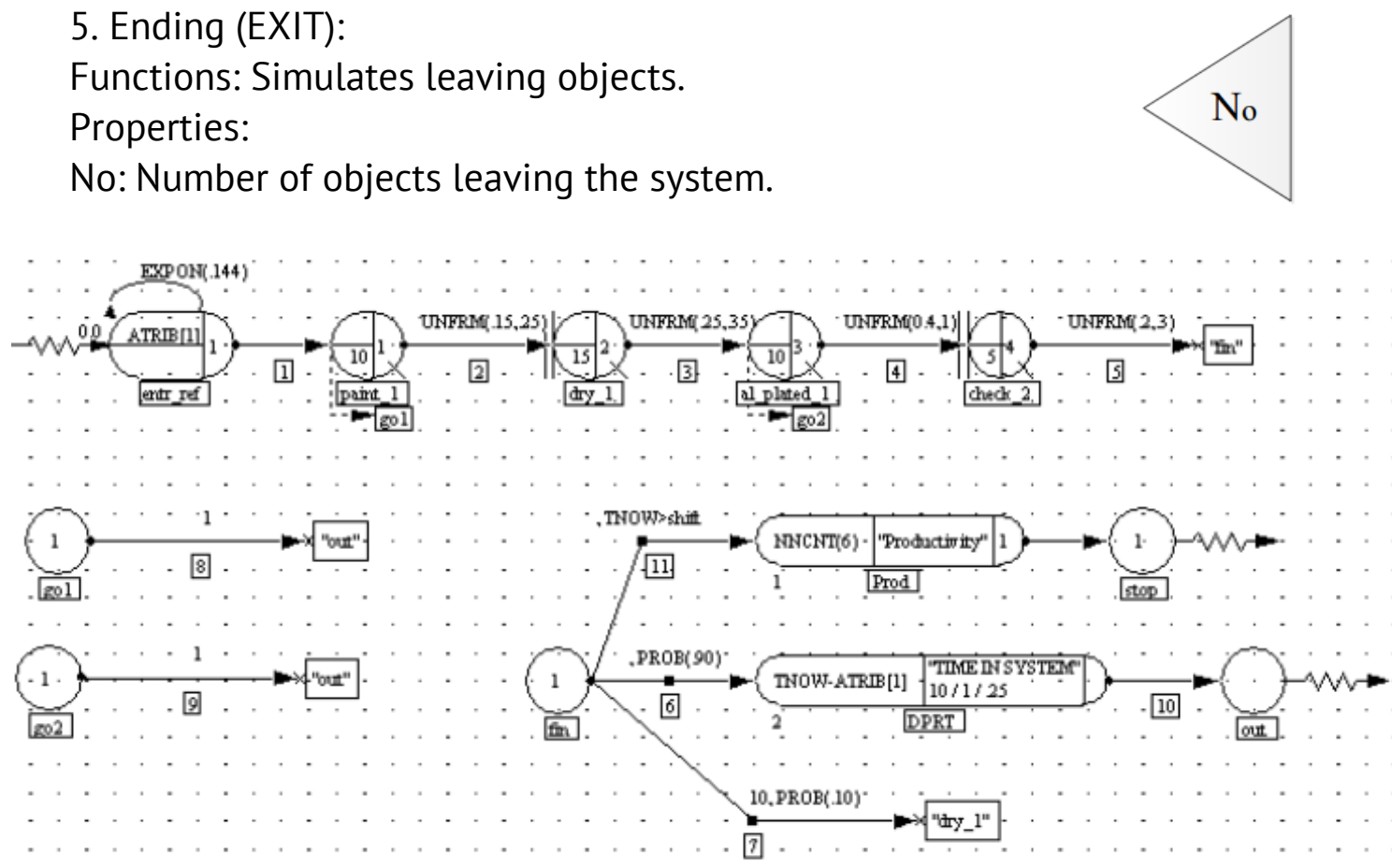

Figure 7. Network model of painting and plating workshop.

Data collection:

The data collected at the present time is as below:

To=EXPON(0.144); T1=0;

TA1=UNFRM $(0.15,0.25) ; \mathrm{TA2}=U N F O R M(0.25,0.35) ; \mathrm{TA3}=U N F O R M(0.4,1)$;

TA4=UNFORM $(0.2,0.3)$;

$\mathrm{CQ} 1=10, \mathrm{CQ} 2=15, \mathrm{CQ} 3=10, \mathrm{CQ} 4=5$

OQ-FIFO

NR1 is a coating machine; NR2 is the drier; NR3 is an aluminum plating machine; NR4 is the inspection worker. 
QR1 is the paint machine number; QR2 is the number of dryers; QR3 is the aluminum number; QR4 is the number of workers checked.

The value of QR1; QR2; QR3; QR4 will depend on the scenarios.

Calculate the number of runs required

In the production workshop, there are the following conditions:

- Working time for one shift: $60 \times 8=480$ minutes.

- The output per shift: 3000 details

- With confidence level: $99 \%$.

The required parameters wil be calculated by running the sample a finite number of times then based on those parameters to calculate the number of runs needed.

With 99\% confidence we follow the Student standard distribution.

We use the formula:

$$
\bar{x}-t_{v-1,1-\alpha} \cdot \frac{s}{\sqrt{5}} \leq \mu \leq \bar{x}+t_{v-1,1-\alpha} \cdot \frac{s}{\sqrt{5}}
$$

$\mathrm{x}$ : Average value

S: Standard deviation

$\mu$ : Average density

$t_{v-1,1-\alpha}$ : Coefficient of the Student standard distribution

Number of runs: 5; Degree of freedom: 4

The value variation: 30 products are equivalent to $1 \%$ of output per shift. We use the following formula to calculate the number of runs needed

$$
\mu \leq \bar{x}+t_{v-1,1-\alpha} \cdot \frac{s}{\sqrt{n}}
$$

So

$$
\begin{gathered}
n \leq\left(t_{v-1,1-\alpha \cdot \frac{s}{\mu-\bar{x}}}\right)^{2} \\
\bar{x}=3274.4
\end{gathered}
$$

$\mathrm{S}=54.848$

$\mathrm{t}_{\mathrm{v}-1,1-\alpha}=3.365$

So $\mathrm{N}=152$ times.

\section{Results and discussion}

The results obtained after using the above parameters.

File Average Length: RUN 1

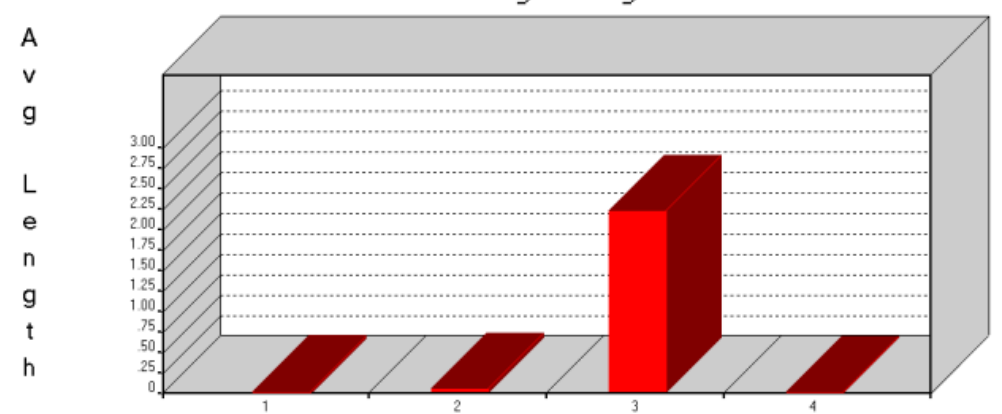

Figure 8. Average queue length. 
File Average Length: RUN 1
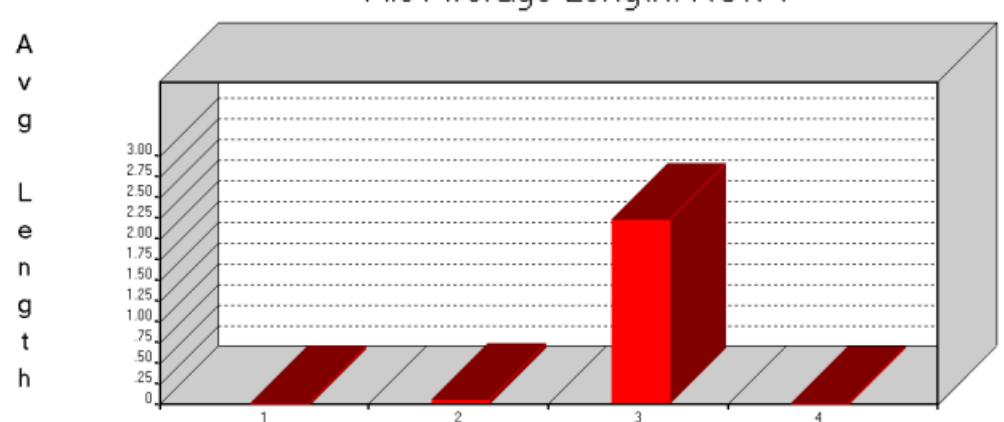

Figure 9. Average waiting time in line.

Service Act. Utilization: RUN 1

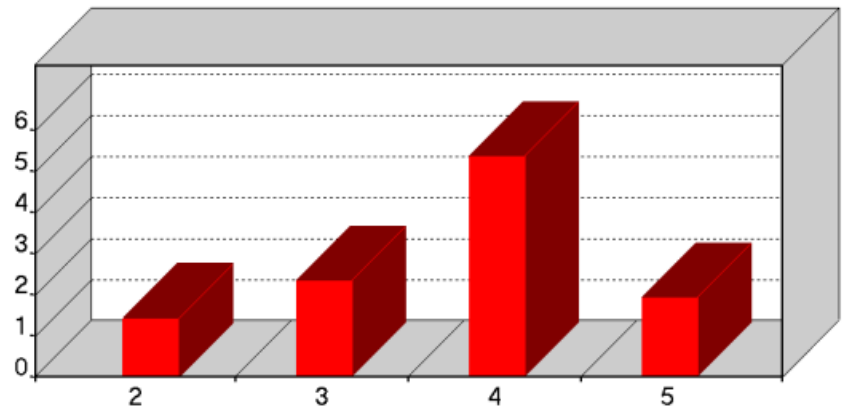

Figure 10. Average resource usage.

Analyze the results obtained about the system with the information in Table 1:

Table 1

Paint shop system status

\begin{tabular}{|c|c|}
\hline $\begin{array}{l}\text { System } \\
\text { information }\end{array}$ & Status \\
\hline Bottlenecks & $\begin{array}{l}\text { The maximum mean queue length was recorded in } \\
\text { action } 3 \text {, in the plating machine area }\end{array}$ \\
\hline $\begin{array}{l}\text { Waiting time in } \\
\text { line }\end{array}$ & Small average waiting time in a queue \\
\hline $\begin{array}{l}\text { Power usage } \\
\text { level }\end{array}$ & $\begin{array}{l}\text { - Maximum power usage is recorded in action } 4 \text { at the } \\
\text { galvanizing machine site. } \\
\text { - In the } 2 \text { nd largest source usage check area recorded in } \\
\text { the } 3 r \text { column of activity in the dryer area. } \\
\text { - In the test area for the third largest level of resource } \\
\text { usage, which is recorded in the column } 5 \text { activity in the } \\
\text { area of the inspected worker. } \\
\text { - Finally, the power usage level is recorded in activity } 2 \\
\text { in the paint machine area. } \\
\text { - In the paint machine area the average value is } 1.402 \\
\text { with the standard distribution value of } 1.17 . \\
\text { - In the drying area, the mean value was } 2.327 \text { with a } \\
\text { standard distribution value of } 1.421 \\
\text { - In the plating machine area, the average value is } 5.344 \\
\text { with a standard distribution value of } 1.222 \\
\text { - At the position of the inspecting worker, the mean is } \\
1.911 \text { with the standard distribution value of } 1.781\end{array}$ \\
\hline
\end{tabular}


A scenario designs for the production process:

From the analysis of the current state of the workshop, the scenario is studied on the use of the source as follows:

- At the coating machine, there are 6 machines, but the actual number of machines used is

$$
n_{c u t}=\bar{x}+3 s=1.402+3 * 1.17=4.912
$$

In $99 \%$ of the cases given as the normal distribution, only 5 out of 6 machines were used to make the product. From the above results it is concluded that reducing the number of paint machines in the paint area does not decrease productivity. Scenarios for the cases are shown in Table 2.

Table 2

\section{Scenario with different number of paint machines}

\begin{tabular}{cc}
\hline Scenario & $\begin{array}{c}\text { Number of paint } \\
\text { machines }\end{array}$ \\
\hline WS2_Paint1 & 1 \\
WS2_Paint2 & 2 \\
WS2_Paint3 & 3 \\
WS2_Paint4 & 4 \\
WS2_Paint5 & 5 \\
\hline
\end{tabular}

The system will simulate more than 152 runs to achieve $\rho 1 \%$ of the time interval of the yield prediction with an average $99 \%$ confidence level.

- In the inspection area, there are 8 workers, but in reality the number of workers needed is

$$
n_{\text {cut }}=\bar{x}+3 s=1.911+3 * 1.781=7.254
$$

In $99 \%$ of the cases given the normal distribution, only 7 out of 8 workers used it to make the product. From the above results it is concluded that reducing the number of workers in the test area does not decrease productivity.

Scenario with different number of workers

\begin{tabular}{cc}
\hline Scenario & Number of workers \\
\hline WS2_CHK1 & 1 \\
WS2_CHK2 & 2 \\
WS2_CHK3 & 3 \\
WS2_CHK4 & 4 \\
WS2_CHK5 & 5 \\
WS2_CHK6 & 6 \\
WS2_CHK7 & 7 \\
\hline
\end{tabular}

Table 3

Improved resource use is the plating machines. All six plating machines are used as follows:

$$
\begin{gathered}
n_{\text {All-plate }}=\bar{x}+\text { z.s } \\
z=\frac{n_{\text {All-plate }}-\bar{x}}{s}=\frac{6-5.344}{1.22}=0.54
\end{gathered}
$$


Given $z=0.54$ regions below the mean curve of 0.2257 leads to the conclusion that most $23 \%$ of cases are using the same $99 \%$ confidence interval. So, in the case productivity can be improved in two ways:

+ Reduce processing time: this problem cannot be done because machines and technology are limited. For these reasons this approach was not considered.

+ Replace plating machines with more productive ones: this option would require a short-term investment to purchase new machines but in the long run will contribute to the company's profits by increasing productivity.

Product processing time here is limited in productivity of test workers with product speed $[0.2 \div 0.3]$ minutes per product. Therefore, this yield has practical value as the number of machines required to keep the yield with the time of [UNFORM, $(0.15,0.35])$.

Table 4

\begin{tabular}{cc} 
Scenario with number of new plating mac \\
\hline Scenario & $\begin{array}{c}\text { Number of plating } \\
\text { machines }\end{array}$ \\
\hline WS2_Al1 & 1 \\
WS2_Al2 & 2 \\
WS2_Al3 & 3 \\
WS2_Al4 & 4 \\
WS2_Al5 & 5 \\
\hline
\end{tabular}

The source usage in the scenarios given in the diagram is as follows:

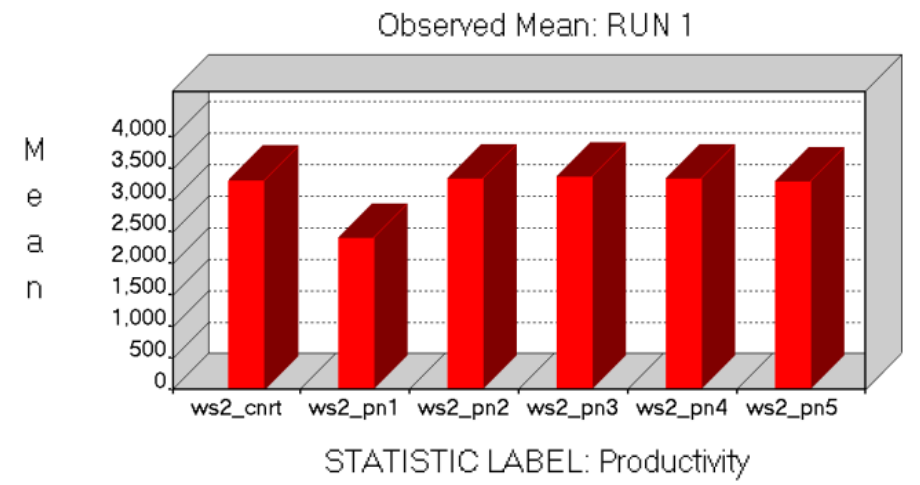

Figure 11. Productivity chart with scenarios with different number of painting machines.

From the above chart, it can be seen that: A steady state of productivity is used with 3 machines. The increase in the number of machines does not increase the productivity, the small change in productivity is obtained due to the mean to shift from the random nature of the mean yield. Therefore, the production line does not reduce productivity if only 3 out of 6 machines are used in the paint machine area.

The productivity charted with different scenarios of the number of workers tested his as.

Through the above chart, it can be seen that: The steady state of productivity is received when the line has 3 inspection workers and the increase in the number of inspection workers does not increase productivity. Small variation in yield is obtained by the mean to shift from the randomness of the mean yield. Thus, the line does not reduce productivity if only 3 out of 8 workers are employed in the test area.

The chart comparing the current scenario and the new scenario has reduced the number of machines and the number of test workers. 


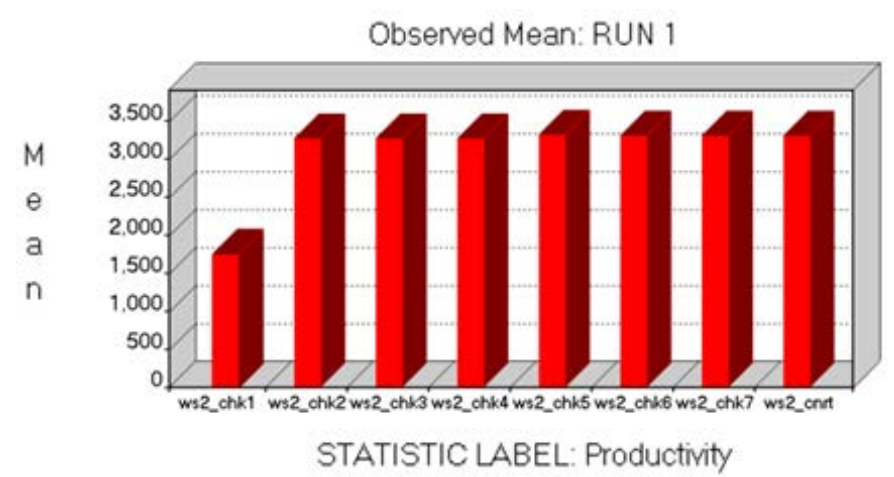

Figure 12. Productivity chart with different scenarios of the number of workers tested.

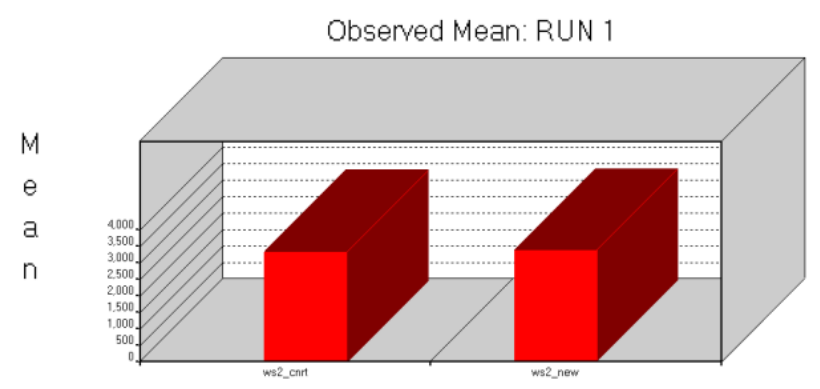

STATISTIC LABEL: Productivity

Figure 13. The chart comparing the current scenario and the new scenario has reduced the number of machines and the number of test workers.

Productivity chart with new number of plating machines for improved equipment management.

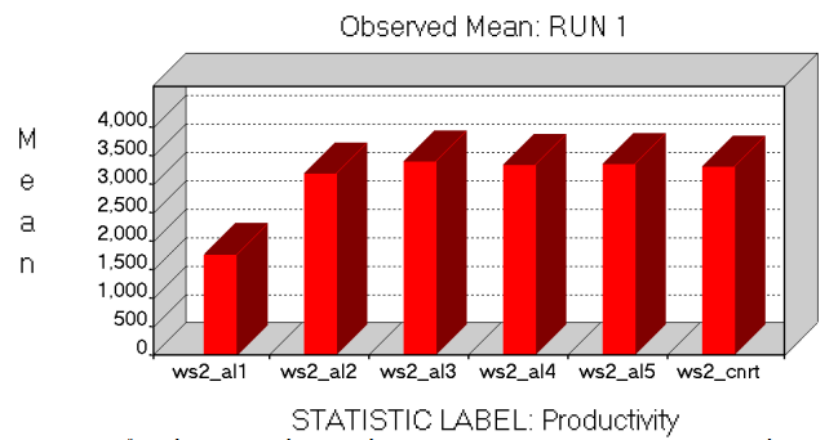

Figure 14. Productivity chart with new number of plating machines for improved equipment management.

The above chart shows that: The steady state of productivity is obtained when the line has 3 plating machines. Increasing the number of machines does not increase productivity, productivity is limited by the number of input part ratios of the workshop. Small variation in yield is obtained by the mean to shift from the randomness of the mean yield.

From the above results it can be seen that the productivity will be suitable when using 3 machines with product speed within the UNFORM limit $[0.15,0.35]$. If you want to increase productivity, the percentage of input details of the workshop must also increase.

\section{Conclusion}

Through this research, the process of modeling simulation and production analysis was carried out in a manufacturing workshop. On that basis, we have analyzed the requirements and designed an experimental model to simulate the operation of the automatic coating and plating line. The model addresses a number of requirements: 
- Visual and general description of the distribution of equipment in the workshop.

- Simulate the activities of the equipment in the chain.

- Simulation helps the manager to come up with the plan to use the equipment effectively, contributing to improving productivity and reducing product costs.

Through this research will be the basis to help production lines to build new or adjust equipment and labor to bring productivity and reduce costs for production.

\section{References}

1. A. Alan B. Pritsker, Introduction to Simulation and SLAM II. 3rd ed., Systems Publishing Corporation, West Lafayette, Indiana, 1986.

2. A. Alan B. Pritsker, O"Reilly, Jean J, Simulation with Visual SLAM /I and Awesim, John Willey \& Sons Inc, US, 1997.

3. A. Alan B. Pritsker, O"Reilly, Jean J, Introduction Simulation SLAM II, John Willey \&Sons Inc, US, 1986.

4. Pristker corporation, Awesim total simulation project support. User's Guide. Symix systems, Inc, 1999.

5. A. Alan B. Pritsker, Jean J. O'Reilly, Simulation with Visual SLAM and AweSim. John Wiley \& Sons, US, 1999.

6. Carrie, A., Simulation of Manufacturing Systems, John Willey \& Sons, Inc, US, 1988.

7. Vantrong Thai, Junsheng Cheng, Optimizing SVM's parameters based on backtracking search optimization algorithm for gear fault diagnosis. Journal of Vibroengineering, 2019. DOI:10.21595/jve.2018.19859.

8. Nguyen Thanh Nhan, Production process improvement in transportation industry in north Vietnam through research and appilication of advanced discrete event simulation modeling techniques. Ph.D thesis, 2008. 